\title{
Picard iterations for nonexpansive and Lipschitz strongly accretive mappings in a real Banach space
}

\author{
Shin Min Kang ${ }^{1}$, Arif Rafiq ${ }^{2}$, Nawab Hussain ${ }^{3}$ and Young Chel Kwun ${ }^{4 *}$
}

"Correspondence:
yckwun@dau.ac.kr
${ }^{4}$ Department of Mathematics,
Dong-A University, Pusan, 614-714,
Korea
Full list of author information is
available at the end of the article

available at the end of the article

\begin{abstract}
We study the convergence of a more general Picard iterative sequence for nonexpansive and Lipschitz strongly accretive mappings in an arbitrary real Banach space. Our results improve the results of Ćirić et al. (Nonlinear Anal. 70(12):4332-4337, 2009).

MSC: 47H06; 47H09; 47J05; 47J25

Keywords: Picard iteration; Lipschitz mapping; nonexpansive mappings; strongly accretive mapping; strongly pseudocontractive mapping; Banach space
\end{abstract}

\section{Introduction and preliminaries}

Let $E$ be a real Banach space with dual $E^{*}$, and $J$ will denote the normalized duality map from $E$ to $2^{E^{*}}$ defined by

$$
J x=\left\{f^{*} \in E^{*}:\left\langle x, f^{*}\right\rangle=\|x\|^{2}=\left\|f^{*}\right\|^{2}\right\},
$$

where $\langle\cdot, \cdot\rangle$ denotes the generalized duality pairing.

Let $T: D(T) \rightarrow E$ be a mapping, where $D(T)$ stands for the domain of $T$.

The mapping $T$ is said to be Lipschitz if there exists $L>0$ such that

$$
\|T x-T y\| \leq L\|x-y\|
$$

for all $x, y \in D(T)$.

If $L=1$ in inequality (1.1), then $T$ is called nonexpansive.

The mapping $T$ is called strongly pseudocontractive if there exists $t>1$ such that

$$
\|x-y\| \leq\|(1+r)(x-y)-r t(T x-T y)\|
$$

for all $x, y \in D(T)$ and $r>0$.

If $t=1$ in inequality (1.2), then $T$ is called pseudocontractive.

As a consequence of the result of Kato [1], it follows from inequality (1.2) that $T$ is strongly pseudocontractive if and only if there exists $j(x-y) \in J(x-y)$ such that

$$
\langle(I-T) x-(I-T) y, j(x-y)| \geq k\|x-y\|^{2}
$$

for all $x, y \in D(T)$, where $k=\frac{t-1}{t} \in(0,1)$. 
Consequently, it follows easily (again from Kato [1] and inequality (1.3)) that $T$ is strongly pseudocontractive if and only if

$$
\|x-y\| \leq\|x-y+s[(I-T-k I) x-(I-T-k I) y]\|
$$

for all $x, y \in D(T)$ and $s>0$.

Closely related to the class of pseudocontractive mappings is the class of accretive operators.

Let $A: D(A) \rightarrow E$ be a mapping.

The mapping $A$ is called accretive if

$$
\|x-y\| \leq\|x-y+s(A x-A y)\|
$$

for all $x, y \in D(A)$ and $s>0$.

Also, as a consequence of Kato [1], this accretive condition can be expressed in terms of the duality mapping as follows:

For each $x, y \in D(A)$, there exists $j(x-y) \in J(x-y)$ such that

$$
\langle A x-A y, j(x-y)\rangle \geq 0
$$

Consequently, inequality (1.2) with $t=1$ yields that $A$ is accretive if and only if $T:=(I-A)$ is pseudocontractive. Furthermore, setting $A:=(I-T)$, it follows from inequality (1.4) that $T$ is strongly pseudocontractive if and only if $(A-k I)$ is accretive, and using (1.5) this implies that $T(=I-A)$ is strongly pseudocontractive if there exists $k \in(0,1)$ such that

$$
\langle A x-A y, j(x-y)\rangle \geq k\|x-y\|^{2}
$$

for all $x, y \in D(A)$.

The mapping $A$ satisfying inequality (1.6) is called strongly accretive. It is then clear that $A$ is strongly accretive if and only if $T:=(I-A)$ is strongly pseudocontractive.

It is worth to mention that considerable research efforts have been devoted, especially within the past long years or so, to developing constructive techniques for the determination of the kernels of accretive operators in Banach spaces (see, e.g., [2-12]). Two wellknown iterative schemes, the Mann iterative scheme (see, e.g., [13]) and the Ishikawa iterative scheme (see, e.g., [14]), have successfully been employed.

In [9], Liu obtained a fixed point of the strictly pseudocontractive mapping as the limit of an iteratively constructed sequence in general Banach spaces.

Theorem 1.1 Let X be a Banach space and let $K$ be a nonempty closed convex and bounded subset of $X$. Let $T: K \rightarrow K$ be Lipschitz (with constant $L \geq 1$ ) and strictly pseudocontractive (i.e., $T$ satisfies inequality (1.4) for all $x, y \in K)$. Let $F(T)=\{x \in X: T x=x\} \neq \emptyset$. For arbitrary $x_{1} \in K$, define the sequence $\left\{x_{n}\right\}$ in $K$ by

$$
x_{n+1}=\left(1-\alpha_{n}\right) x_{n}+\alpha_{n} T x_{n},
$$


where $\left\{\alpha_{n}\right\}$ is a sequence in $(0,1]$ satisfying

$$
\sum_{n=1}^{\infty} \alpha_{n}=\infty, \quad \alpha_{n} \rightarrow 0
$$

Then $\left\{x_{n}\right\}$ converges strongly to $q \in F(T)$ and $F(T)$ is a singleton.

By generalizing the results of Liu [9], Sastry and Babu [11] proved the following results.

Theorem 1.2 Let $X$ be a Banach space and let $K$ be a nonempty closed convex and bounded subset of $X$. Let $T: K \rightarrow K$ be Lipschitz (with constant $L \geq 0$ ) and strictly pseudocontractive (i.e., T satisfies inequality (1.4) for all $x, y \in K)$. Suppose that $\left\{\alpha_{n}\right\}_{n \in \mathbb{N}}$ is a sequence in $(0,1]$ such that for some $\eta \in(0, k)$ and for all $n \in \mathbb{N}$,

$$
\alpha_{n} \leq \frac{k-\eta}{(L+1)(L+2-k)}, \quad \sum_{n=1}^{\infty} \alpha_{n}=\infty
$$

Fix $x_{1} \in K$. Define the sequence $\left\{x_{n}\right\}_{n \in \mathbb{N}}$ in $K$ by

$$
x_{n+1}:=\left(1-\alpha_{n}\right) x_{n}+\alpha_{n} T x_{n}, \quad n \in \mathbb{N} .
$$

Then there exists $\left\{\beta_{n}\right\}_{n \in \mathbb{N}}$, a sequence in $(0,1)$ with each $\beta_{n} \geq \frac{\eta}{1+k} \alpha_{n}$, such that

$$
\left\|x_{n+1}-q\right\| \leq \prod_{j=1}^{n}\left(1-\beta_{j}\right)\left\|x_{1}-q\right\|, \quad n \in \mathbb{N}
$$

In particular, $\left\{x_{n}\right\}_{n \in \mathbb{N}}$ converges strongly to $q \in K$ and $q$ is the unique fixed point of $T$.

In $[15,16]$, Chidume mentioned that the Mann and Ishikawa iteration schemes are global and their rate of convergence is generally of the order $O\left(n^{-\frac{1}{2}}\right)$. Also, it is well-known that for an operator $U$, the classical iterative sequence $x_{n+1}=U x_{n}, x_{0} \in D(U)$ (called the Picard iterative sequence) converges and is preferred in comparison to the Mann or the Ishikawa sequences since it requires less computations; and moreover, its rate of convergence is always at least as fast as that of a geometric progression.

In $[15,16]$, Chidume proved the following results.

Theorem 1.3 Let $E$ be an arbitrary real Banach space, let $A: E \rightarrow E$ be a Lipschitz (with constant $L>0)$ and strongly accretive mapping with strong accretivity constant $k \in(0,1)$. Let $x^{*}$ denote a solution of the equation $A x=0$. Set $\epsilon:=\frac{1}{2}\left(\frac{k}{1+L(3+L-k)}\right)$ and define $A_{\epsilon}: E \rightarrow E$ by $A_{\epsilon} x:=x-\epsilon A x$ for each $x \in E$. For arbitrary $x_{0} \in E$, define the sequence $\left\{x_{n}\right\}_{n=0}^{\infty}$ in $E$ by

$$
x_{n+1}=A_{\epsilon} x_{n}, \quad n \geq 0 .
$$

Then $\left\{x_{n}\right\}_{n=0}^{\infty}$ converges strongly to $x^{*}$ with

$$
\left\|x_{n+1}-x^{*}\right\| \leq \delta^{n}\left\|x_{0}-x^{*}\right\|
$$

where $\delta=\left(1-\frac{1}{2} k \epsilon\right) \in(0,1)$. Moreover, $x^{*}$ is unique. 
Corollary 1.4 Let $E$ be an arbitrary real Banach space and let $K$ be a nonempty convex subset of E. Let $T: K \rightarrow K$ be Lipschitz (with constant $L>0$ ) and strongly pseudocontractive (i.e., $T$ satisfies inequality (1.4) for all $x, y \in K)$. Assume that $T$ has a fixed point $x^{*} \in K$. Set $\epsilon_{0}:=\frac{1}{2}\left(\frac{k}{1+L(3+L-k)}\right)$ and define $T_{\epsilon_{0}}: K \rightarrow K$ by $T_{\epsilon_{0}} x=\left(1-\epsilon_{0}\right) x+\epsilon_{0}$ Tx for each $x \in K$. For arbitrary $x_{0} \in K$, define the sequence $\left\{x_{n}\right\}_{n=0}^{\infty}$ in $K$ by

$$
x_{n+1}=T_{\epsilon_{0}} x_{n}, \quad n \geq 0 .
$$

Then $\left\{x_{n}\right\}_{n=0}^{\infty}$ converges strongly to $x^{*}$ with

$$
\left\|x_{n+1}-x^{*}\right\| \leq \delta^{n}\left\|x_{0}-x^{*}\right\|,
$$

where $\delta:=\left(1-\frac{1}{2} k \epsilon_{0}\right) \in(0,1)$. Moreover, $x^{*}$ is unique.

Recently, Cirić et al. [17] improved the results of Chidume [15, 16], Liu [9] and Sastry and Babu [11].

We study the convergence of a more general Picard iterative sequence for nonexpansive and Lipschitz strongly accretive mappings in an arbitrary real Banach space. Our results improve the results of Ćirić et al. [17].

\section{Main results}

In the following theorems, $L>0$ will denote the Lipschitz constant of the operator $A$ and $k>0$ will denote the strong accretivity constant of $A$ (as in inequality (1.6)). Furthermore, in [17], $\epsilon>0$ is defined by

$$
\epsilon:=\frac{k-\eta}{L(2+L)}, \quad \eta \in(0, k)
$$

With these notations, we prove the following theorem.

Theorem 2.1 Let $E$ be an arbitrary real Banach space, let $A^{\prime}: E \rightarrow E$ be nonexpansive and let $A: E \rightarrow E$ be a Lipschitz strongly accretive mapping with strong accretivity constant $k \in(0,1)$. Let $x^{*}$ denote a solution of the system $A^{\prime} x=0=A x$. Define $A_{\epsilon}: E \rightarrow E$ by $A_{\epsilon} x:=$ $x-\epsilon A x-A^{\prime}(x-\epsilon A x)$ for each $x \in E$. For arbitrary $x_{0} \in E$, define the sequence $\left\{x_{n}\right\}_{n=0}^{\infty}$ in $E$ by

$$
x_{n+1}=A_{\epsilon} x_{n}, \quad n \geq 0 .
$$

Then $\left\{x_{n}\right\}_{n=0}^{\infty}$ converges strongly to $x^{*}$ with

$$
\left\|x_{n+1}-x^{*}\right\| \leq \rho^{n}\left\|x_{0}-x^{*}\right\|,
$$

where $\rho=\left(1-\frac{k-\eta}{k(k-\eta)+L(2+L)} \eta\right) \in(0,1)$. Thus, the choice $\eta=\frac{k}{2}$ yields $\rho=1-\frac{k^{2}}{2[k+2 L(2+L)]}$. Moreover, $x^{*}$ is unique.

Proof Let $S=I-A^{\prime}$ and $T=I-A$, where $I$ denotes the identity mapping on $E$. Observe that $A^{\prime} x^{*}=0=A x^{*}$ if and only if $x^{*}$ is a common fixed point of $S$ and $T$. Moreover, $T$ is 
strongly pseudocontractive since $A$ is strongly accretive. Therefore, $T$ satisfies inequality (1.4) for all $x, y \in E$ and $s>0$. Furthermore, the recursion formula $x_{n+1}=A_{\epsilon} x_{n}$ becomes

$$
\left\{\begin{array}{l}
x_{n+1}=S y_{n}, \\
y_{n}=(1-\epsilon) x_{n}+\epsilon T x_{n}, \quad n \geq 0 .
\end{array}\right.
$$

Observe that

$$
x^{*}=(1+\epsilon) x^{*}+\epsilon(I-T-k I) x^{*}-(1-k) \epsilon x^{*},
$$

and from recursion formula (2.1) we get

$$
\begin{aligned}
x_{n}= & (1+\epsilon) y_{n}+\epsilon(I-T-k I) y_{n}-(1-k) \epsilon y_{n} \\
& +\epsilon\left(x_{n}-y_{n}\right)+\epsilon\left(T y_{n}-T x_{n}\right) \\
= & (1+\epsilon) y_{n}+\epsilon(I-T-k I) y_{n}-(1-k) \epsilon y_{n} \\
& +\epsilon^{2}\left(x_{n}-T x_{n}\right)+\epsilon\left(T y_{n}-T x_{n}\right),
\end{aligned}
$$

so that

$$
\begin{aligned}
x_{n}-x^{*}= & (1+\epsilon)\left(y_{n}-x^{*}\right)+\epsilon\left[(I-T-k I) y_{n}-(I-T-k I) x^{*}\right] \\
& -(1-k) \epsilon\left(y_{n}-x^{*}\right)+\epsilon^{2}\left(x_{n}-T x_{n}\right)+\epsilon\left(T y_{n}-T x_{n}\right) .
\end{aligned}
$$

This implies, using inequality (1.4) with $s=\frac{\epsilon}{1+\epsilon}$ and $y=x^{*}$, that

$$
\begin{aligned}
\left\|x_{n}-x^{*}\right\| \geq & (1+\epsilon)\left[\left\|\left(y_{n}-x^{*}\right)+\frac{\epsilon}{1+\epsilon}\left[(I-T-k I) y_{n}-(I-T-k I) x^{*}\right]\right\|\right] \\
& -(1-k) \epsilon\left\|y_{n}-x^{*}\right\|-\epsilon^{2}\left\|x_{n}-T x_{n}\right\|-\epsilon\left\|T y_{n}-T x_{n}\right\| \\
\geq & (1+\epsilon)\left\|y_{n}-x^{*}\right\|-(1-k) \epsilon\left\|y_{n}-x^{*}\right\|-\epsilon^{2}\left\|x_{n}-T x_{n}\right\|-\epsilon\left\|T y_{n}-T x_{n}\right\| \\
= & (1+k \epsilon)\left\|y_{n}-x^{*}\right\|-\epsilon^{2}\left\|x_{n}-T x_{n}\right\|-\epsilon\left\|T y_{n}-T x_{n}\right\| .
\end{aligned}
$$

Observe that

$$
\left\|x_{n}-T x_{n}\right\| \leq L\left\|x_{n}-x^{*}\right\|, \quad\left\|T y_{n}-T x_{n}\right\| \leq \epsilon L(1+L)\left\|x_{n}-x^{*}\right\|,
$$

so that

$$
\left\|x_{n}-x^{*}\right\| \geq(1+k \epsilon)\left\|y_{n}-x^{*}\right\|-\epsilon[1-k+\epsilon L(2+L)]\left\|x_{n}-x^{*}\right\|,
$$

which implies that

$$
\left\|y_{n}-x^{*}\right\| \leq \frac{1+\epsilon[1-k+\epsilon L(2+L)]}{1+k \epsilon}\left\|x_{n}-x^{*}\right\|,
$$


and we have

$$
\begin{aligned}
\rho & =\frac{1+\epsilon[1-k+\epsilon L(2+L)]}{1+k \epsilon} \\
& =1-\frac{\epsilon}{1+k \epsilon}[k-\epsilon L(2+L)] \\
& =1-\frac{\epsilon}{1+k \epsilon} \eta \\
& =1-\frac{k-\eta}{k(k-\eta)+L(2+L)} \eta .
\end{aligned}
$$

From (2.5) and (2.6), we get

$$
\left\|y_{n}-x^{*}\right\| \leq \rho\left\|x_{n}-x^{*}\right\| \leq \cdots \leq \rho^{n}\left\|x_{0}-x^{*}\right\| .
$$

Hence, $y_{n} \rightarrow x^{*}$ as $n \rightarrow \infty$. Finally, by (2.1) and (2.7), we obtain

$$
\begin{aligned}
\left\|x_{n+1}-x^{*}\right\| & =\left\|S y_{n}-x^{*}\right\| \\
& \leq\left\|y_{n}-x^{*}\right\| \leq \rho^{n}\left\|x_{0}-x^{*}\right\| \\
& \rightarrow 0 \quad \text { as } n \rightarrow \infty .
\end{aligned}
$$

Uniqueness follows from the strong accretivity property of $A$. This completes the proof.

The following is an immediate corollary of the above theorem.

Corollary 2.2 Let $E$ be an arbitrary real Banach space and let $K$ be a nonempty closed convex subset of E. Let $S: K \rightarrow K$ be nonexpansive and $T: K \rightarrow K$ be Lipschitz (with constant $L>0$ ) and strongly pseudocontractive (i.e., $T$ satisfies inequality (1.4) for all $x, y \in K$ ). Assume that $S$ and $T$ have a common fixed point $x^{*}$ in $K$. Set $\epsilon_{0}:=\frac{k-\eta}{L(2+L)}, \eta \in(0, k)$ and define $H_{\varepsilon_{0}}: K \rightarrow K$ by $H_{\varepsilon_{0}} x=S\left(\left(1-\varepsilon_{0}\right) x+\varepsilon_{0} T x\right)$ for each $x \in K$. For arbitrary $x_{0} \in E$, define the sequence $\left\{x_{n}\right\}_{n=0}^{\infty}$ in $E$ by

$$
x_{n+1}=H_{\epsilon_{0}} x_{n}, \quad n \geq 0 .
$$

Then $\left\{x_{n}\right\}_{n=0}^{\infty}$ converges strongly to $x^{*}$ with

$$
\left\|x_{n+1}-x^{*}\right\| \leq \rho^{n}\left\|x_{0}-x^{*}\right\|
$$

where $\rho=\left(1-\frac{k-\eta}{k(k-\eta)+L(2+L)} \eta\right) \in(0,1)$. Moreover, $x^{*}$ is unique.

Proof Observe that $x^{*}$ is a common fixed point of $S$ and $T$, then it is a fixed point of $H_{\epsilon_{0}}$. Furthermore, recursion formula (2.1) simplifies to the formula

$$
\left\{\begin{array}{l}
x_{n+1}=S y_{n}, \\
y_{n}=\left(1-\epsilon_{0}\right) x_{n}+\epsilon_{0} T x_{n}, \quad n \geq 0,
\end{array}\right.
$$


which is similar to (2.1). Following the method of computations as in the proof of Theorem 2.1, we obtain

$$
\begin{aligned}
\left\|x_{n+1}-x^{*}\right\| & =\left\|S y_{n}-x^{*}\right\| \\
& \leq\left\|y_{n}-x^{*}\right\| \\
& \leq \frac{1+\epsilon_{0}\left[1-k+\epsilon_{0} L(2+L)\right]}{1+k \epsilon_{0}}\left\|x_{n}-x^{*}\right\| \\
& \leq\left(1-\frac{k-\eta}{k(k-\eta)+L(2+L)} \eta\right)\left\|x_{n}-x^{*}\right\| .
\end{aligned}
$$

Set $\rho_{0}=1-\frac{k-\eta}{k(k-\eta)+L(2+L)} \eta$, then from (2.9) we obtain

$$
\left\|x_{n+1}-x^{*}\right\| \leq \rho_{0}\left\|x_{n}-x^{*}\right\| \leq \cdots \leq \rho_{0}^{n}\left\|x_{0}-x^{*}\right\| \rightarrow 0 \quad \text { as } n \rightarrow \infty .
$$

This completes the proof.

From Theorem 2.1 and Corollary 2.2, we reduce recent results in [17] to the following.

Theorem 2.3 Let $E$ be an arbitrary real Banach space, let $A: E \rightarrow E$ be a Lipschitz (with constant $L>0)$ and strongly accretive mapping with strong accretivity constant $k \in(0,1)$. Let $x^{*}$ denote a solution of the equation $A x=0$. Set $\epsilon:=\frac{k-\eta}{L(2+L)}, \eta \in(0, k)$ and define $A_{\varepsilon}$ : $E \rightarrow E$ by $A_{\varepsilon} x:=x-\varepsilon A x$ for each $x \in E$. For arbitrary $x_{0} \in E$, define the sequence $\left\{x_{n}\right\}_{n=0}^{\infty}$ in Eby

$$
x_{n+1}=A_{\varepsilon} x_{n}, \quad n \geq 0 .
$$

Then $\left\{x_{n}\right\}_{n=0}^{\infty}$ converges strongly to $x^{*}$ with

$$
\left\|x_{n+1}-x^{*}\right\| \leq \theta^{n}\left\|x_{0}-x^{*}\right\|
$$

where $\theta=\left(1-\frac{k-\eta}{k(k-\eta)+L(2+L)} \eta\right) \in(0,1)$. Thus the choice $\eta=\frac{k}{2}$ yields $\theta=1-\frac{k^{2}}{2\left[k^{2}+2 L(2+L)\right]}$. Moreover, $x^{*}$ is unique.

Corollary 2.4 Let $E$ be an arbitrary real Banach space and let $K$ be a nonempty convex subset of E. Let $T: K \rightarrow K$ be Lipschitz (with constant $L>0$ ) and strongly pseudocontractive (i.e., $T$ satisfies inequality (1.4) for all $x, y \in K)$. Assume that $T$ has a fixed point $x^{*} \in K$. Set $\varepsilon_{0}:=\frac{k-\eta}{L(2+L)}, \eta \in(0, k)$ and define $T_{\varepsilon_{0}}: K \rightarrow K$ by $T_{\varepsilon_{0}} x=\left(1-\varepsilon_{0}\right) x+\varepsilon_{0}$ Tx for each $x \in K$. For arbitrary $x_{0} \in K$, define the sequence $\left\{x_{n}\right\}_{n=0}^{\infty}$ in $K$ by

$$
x_{n+1}=T_{\varepsilon_{0}} x_{n}, \quad n \geq 0 .
$$

Then $\left\{x_{n}\right\}_{n=0}^{\infty}$ converges strongly to $x^{*}$ with

$$
\left\|x_{n+1}-x^{*}\right\| \leq \theta^{n}\left\|x_{0}-x^{*}\right\|
$$

where $\theta:=\left(1-\frac{k-\eta}{k(k-\eta)+L(2+L)} \eta\right) \in(0,1)$. Moreover, $x^{*}$ is unique. 


\section{Competing interests}

The authors declare that they have no competing interests.

\section{Authors' contributions}

All authors read and approved the final manuscript.

\section{Author details}

${ }^{1}$ Department of Mathematics and RINS, Gyeongsang National University, Jinju, 660-701, Korea. ${ }^{2}$ School of CS and Mathematics, Hajvery University, 43-52 Industrial Area, Gulberg-III, Lahore, 54660, Pakistan. ${ }^{3}$ Department of Mathematics, King Abdulaziz University, P.O. Box 80203, Jeddah, 21589, Saudi Arabia. ${ }^{4}$ Department of Mathematics, Dong-A University, Pusan, 614-714, Korea.

\section{Acknowledgements}

This study was supported by research funds from Dong-A University.

Received: 29 May 2013 Accepted: 25 June 2013 Published: 10 July 2013

\section{References}

1. Kato, T: Nonlinear semigroups and evolution equations. J. Math. Soc. Jpn. 19, 508-520 (1967)

2. Chidume, CE: Iterative approximation of fixed points of Lipschitz strictly pseudocontractive mappings. Proc. Am. Math. Soc. 99, 283-288 (1987)

3. Chidume, CE: An iterative process for nonlinear Lipschitz strongly accretive mappings in $L_{p}$ spaces. J. Math. Anal. Appl. 151, 453-461 (1990)

4. Deimling, K: Nonlinear Functional Analysis. Springer, Berlin (1985)

5. Deng, L: On Chidume's open problem. J. Math. Anal. Appl. 174, 441-449 (1991)

6. Deng, L: An iterative process for nonlinear Lipschitz and strongly accretive mappings in uniformly convex and uniformly smooth Banach spaces. Acta Appl. Math. 32, 183-196 (1993)

7. Deng, L: Iteration processes for nonlinear Lipschitzian strongly accretive mappings in $L_{p}$ spaces. J. Math. Anal. Appl. $188,128-140(1994)$

8. Deng, L, Ding, XP: Iterative approximation of Lipschitz strictly pseudocontractive mappings in uniformly smooth spaces. Nonlinear Anal. 24, 981-987 (1995)

9. Liu, L: Approximation of fixed points of a strictly pseudocontractive mapping. Proc. Am. Math. Soc. 125, 1363-1366 (1997)

10. Liu, QH: The convergence theorems of the sequence of Ishikawa iterates for hemicontractive mappings. J. Math. Anal. Appl. 148, 55-62 (1990)

11. Sastry, WR, Babu, GVR: Approximation of fixed points of strictly pseudocontractive mappings on arbitrary closed, convex sets in a Banach space. Proc. Am. Math. Soc. 128, 2907-2909 (2000)

12. Weng, XL: Fixed point iteration for local strictly pseudocontractive mapping. Proc. Am. Math. Soc. 113, 727-731 (1991)

13. Mann, WR: Mean value methods in iteration. Proc. Am. Math. Soc. 4, 506-510 (1953)

14. Ishikawa, S: Fixed point by a new iteration method. Proc. Am. Math. Soc. 44, 147-150 (1974)

15. Chidume, CE: Picard iteration for strongly accretive and strongly pseudocontractive Lipschitz maps. ICTP Preprint no. IC2000098

16. Chidume, CE: Iterative algorithms for nonexpansive mappings and some of their generalizations. In: Nonlinear Analysis and Applications: to V. Lakshmikantam on His 80th Birthday. Vol. 1,2, pp. 383-429. Kluwer Academic, Dordrecht (2003)

17. Ćirić, LB, Rafiq, A, Cakić, N: On Picard iterations for strongly accretive and strongly pseudo-contractive Lipschitz mappings. Nonlinear Anal. 70, 4332-4337 (2009)

\section{Submit your manuscript to a SpringerOpen ${ }^{\circ}$ journal and benefit from:}

- Convenient online submission

Rigorous peer review

- Immediate publication on acceptance

- Open access: articles freely available online

- High visibility within the field

- Retaining the copyright to your article 